\title{
Efficient thermal infrared emitter with high radiant power
}

\author{
Marco Schossig, Tobias Ott, Sarah Hüller, Volkmar Norkus, Gerald Gerlach \\ Technische Universität Dresden, Institute for Solid-State Electronics, 01062 Dresden, Germany \\ marco.schossig@tu-dresden.de
}

\begin{abstract}
:
Sensitive and selective gas measurements are crucial for a large variety of applications, e.g. explosion protection. Optical gas detection is usually based on the gas' absorption of infrared radiation (IR). It is the leading technique in terms of accuracy, reliability, and economic efficiency. Since most gas measurements are made in the two wavelength ranges of $(3 \ldots 5) \mu \mathrm{m}$ and $(8 \ldots 14) \mu \mathrm{m}$, a broadband IR source is necessary. In this paper, we report on a novel thermal IR emitter with high radiant power and near-blackbody emission characteristic that can be modulated electrically. The layout of the IR source had been optimized by use of FEM simulations in order to get an adequate electrical resistance as well as a homogeneous temperature distribution and a minimum deflection of the radiating element. Due to its very low thermal mass and the excellent thermal insulation from the heat sink, the electrical power consumption is very low. Operating temperatures of up to $1400 \mathrm{~K}$ are possible so that the fabricated IR source features a very high radiant power. Its application in gas analysis will improve the performance and efficiency of gas measurement systems.
\end{abstract}

Key words: gas analysis, gas measurement, infrared radiation, infrared source, thermal emitter

\section{Introduction}

Explosion hazards mostly arise from flammable gases and vapours. Instead of avoiding their ignition by explosion protection measurements it may be preferable to detect them before they become ignitable. Depending on the application different measuring principles for the detection of gases and vapours can be used, e.g. electrochemical sensors [1], semiconductor sensors [2], and point or open-path infrared gas detectors [3].

Optical gas detection by use of infrared radiation (IR) is the leading technique in terms of accuracy, reliability, and economic efficiency. Since most gas measurements are made in the two wavelength ranges of $(3 \ldots 5) \mu \mathrm{m}$ and (8 ... 14) $\mu \mathrm{m}$, a broadband IR source with nearblackbody emission characteristic is necessary. For reliable measurements the generation of a stable spectrum of infrared radiation is crucial. Very low detection limits can be realized by a long optical path, where more gas molecules are involved in the IR-absorption. But this requires a very powerful IR source.

According to Planck's law of thermal radiation the spectrum of a thermal emitter with blackbody characteristic depends only on its temperature $T$ [4]. The totally emitted radiant power $P$ is given by the Stefan-Boltzmann law

$P=\varepsilon \cdot \sigma \cdot A \cdot T^{4}$ with emissivity $\varepsilon$, Stefan-Boltzmann constant $\sigma$, and the radiating area $A$. Consequently, a high radiation power is mainly realized by a high operating temperature of the thermal emitter but also by a high and wavelength-independent emissivity of infrared radiation.

Especially portable gas sensing devices require a low energy consumption of the IR source. For this reason, the radiating element has to be optimized regarding a very low thermal mass and an excellent thermal insulation.

\section{Design and technology}

In [5] and [6] we reported on a novel IR absorber based on a nanostructured NiCr-alloy that features a very high and spectrally homogeneous absorptivity. From Kirchhoff's law [4]

$\alpha(\lambda, T)=\varepsilon(\lambda, T)$

it is known that for the maintenance of thermal equilibrium the absorptivity $\alpha$ at wavelength $\lambda$ and temperature $T$ must be equal to the emissivity $\varepsilon$. Therefore, good absorbers are also good emitters. Since $\mathrm{NiCr}$ is one of the most used materials in heating elements, for instance in toasters and hair-dryers, we investigated the application of the nanostructured $\mathrm{NiCr}$ absorber (Fig. 1) in thermal infrared emitters to increase their emittance. 


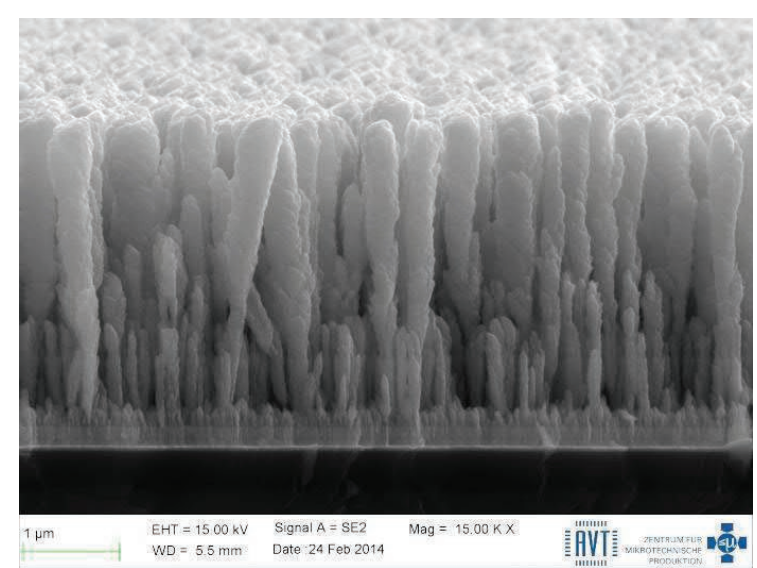

Fig. 1: SEM picture of a nanostructured $\mathrm{NiCr}$ absorber

By depositing the nanostructured $\mathrm{NiCr}$ layer on a thin $\mathrm{NiCr}$ foil the high-temperature stability of the nanostructures could be verified with a first prototype of a thermal IR emitter in a TO-39 package (Fig. 2). Thereby, operation at air causes an oxidation of the surface. The speed of oxidation and the thickness of the oxide are increased with increasing temperature. As a consequence, the spectral emissivity of the nanostructured surface is affected. In contrast, an operation in an inert gas atmosphere does not change its optical properties even at very high temperatures.

The layout of the first prototype has some disadvantages. First, it has a very low electrical resistance of about $1 \Omega$ which is not practicable to use. In addition, the temperature distribution is not homogeneous as it can be seen from Fig. 2. Finally, it has a relatively high power consumption of about $5 \mathrm{~W}$ due to its high emitting area and the poor thermal insulation of the radiating element.

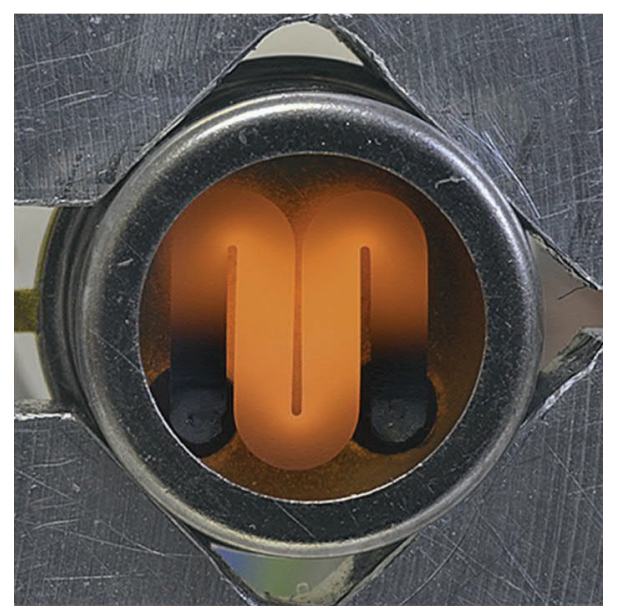

Fig. 2: First prototype of a thermal infrared emitter with nanostructured high-emissivity layer operating at about $1170 \mathrm{~K}$.

In order to optimize the layout and the contacts of the radiating element we used FEM simulations. As a result we found a spiral shape to be optimal regarding an adequate electrical resistance, a homogeneous temperature distribution and a minimum deflection of the radiating element as well as minimum power consumption (Fig. 3). On this basis we fabricated new prototypes. By reducing the thickness of the radiating element to about $10 \mu \mathrm{m}$, a direct electrical modulation of the emitted radiation becomes possible. This is an important feature for its application in gas sensing devices because the most commonly used pyroelectric IR detectors are only sensitive to AC signals. Accordingly, it eliminates the use of a mechanical chopper for radiation modulation and, therefore, allows more compact gas sensing devices.

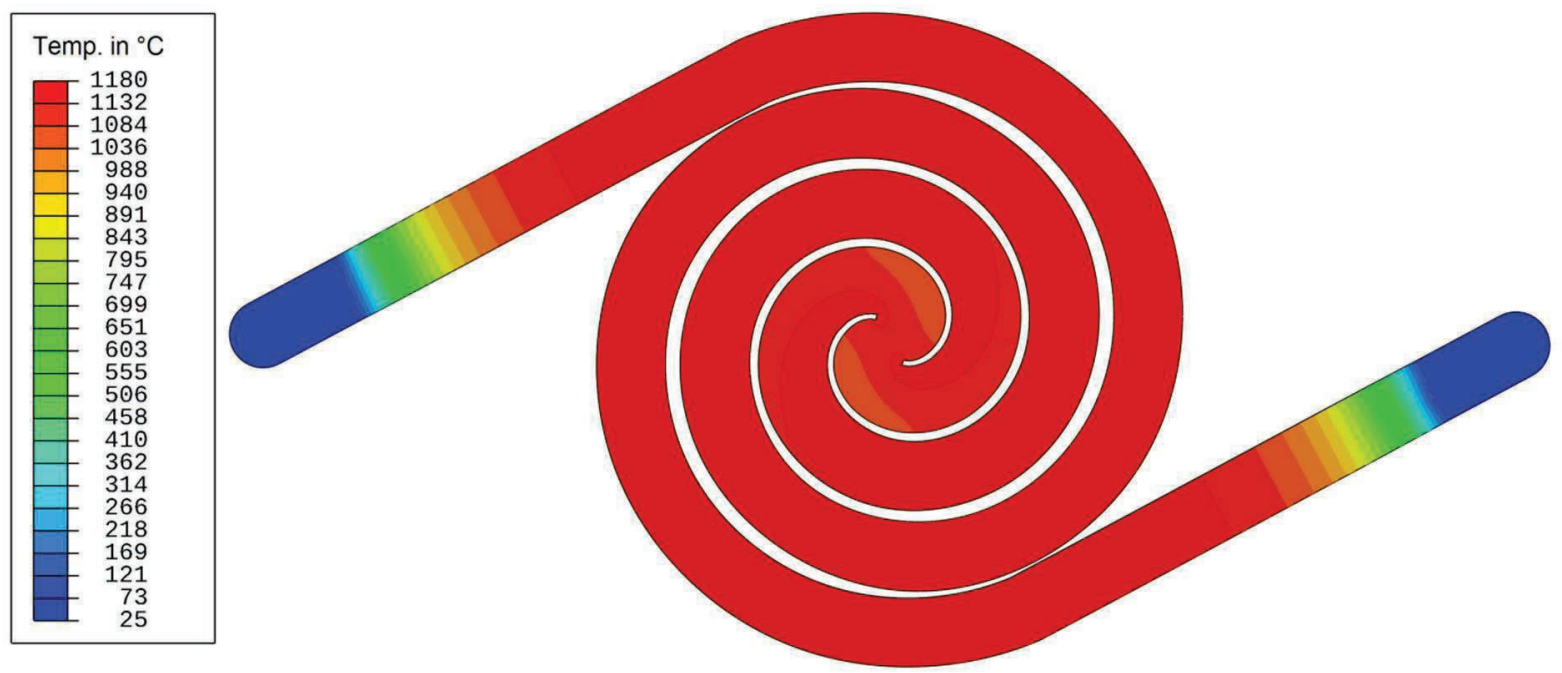

Fig. 3: Optimized layout of the radiating element showing an excellent homogeneous temperature distribution 


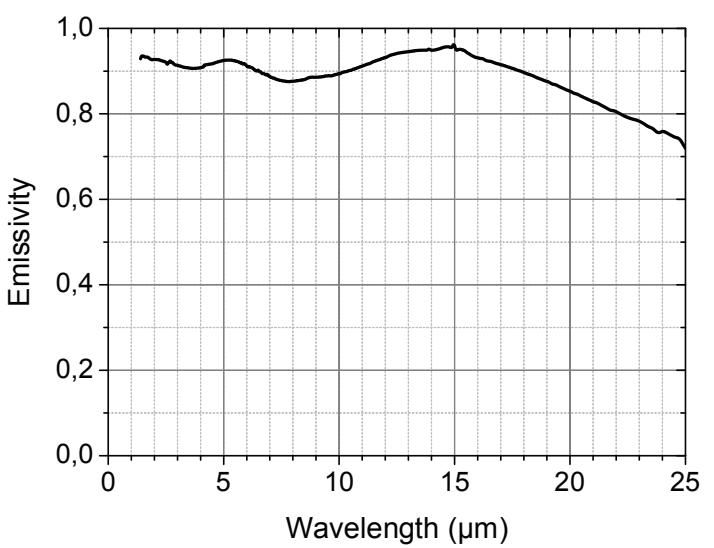

Fig. 4: Measured spectral emissivity of the novel thermal infrared emitters with spiral filament

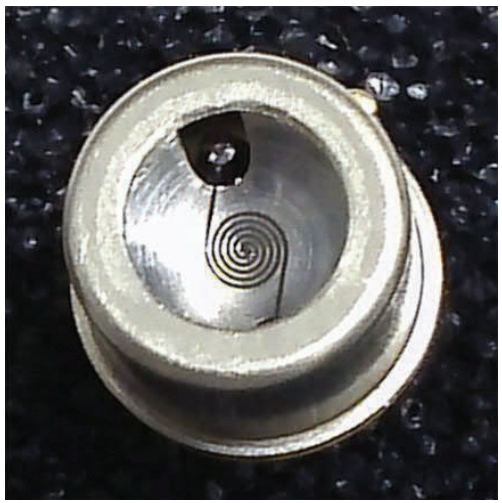

Fig. 5: Prototype of a thermal infrared emitter with near-blackbody emission characteristic in TO-39 housing

The spiral radiating elements were fabricated by ion beam etching of a thin $\mathrm{NiCr}$ foil and have a radiating area of about $2.8 \mathrm{~mm}^{2}$. Both sides are coated with a $2 \mu \mathrm{m}$ thick nanostructured layer of high emissivity (Fig. 4). The radiating elements are hermetically sealed into common TO-39 housings with a krypton atmosphere (Fig. 5). In order to use the backside-emitted radiation a reflector is integrated into the TO-39 housing. In this way, a $70 \%$ higher signal can be detected.

\section{Experimental results}

The electrical and optical properties of the new prototypes were studied. We found an adequate electrical cold resistance of about $31 \Omega \pm 3 \Omega$ and a slightly higher hot resistance of about $33 \Omega \pm 3 \Omega$ (Fig. 6 ). Accordingly, the emitters can be operated at $3.3 \mathrm{~V}$, a common voltage used in portable devices. In this case, the mean electrical power consumption is $330 \mathrm{~mW} \pm 20 \mathrm{~mW}$ with an operating temperature of $970 \mathrm{~K}$ (Fig. 7). Comparable thin-film emitters reach only a maximum temperature of about $800 \mathrm{~K}$ at the same electrical input power.

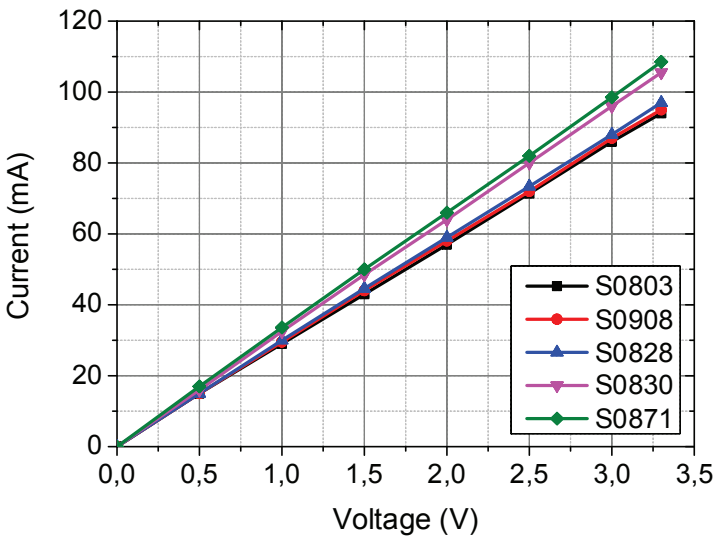

Fig. 6: Measured current-voltage characteristic

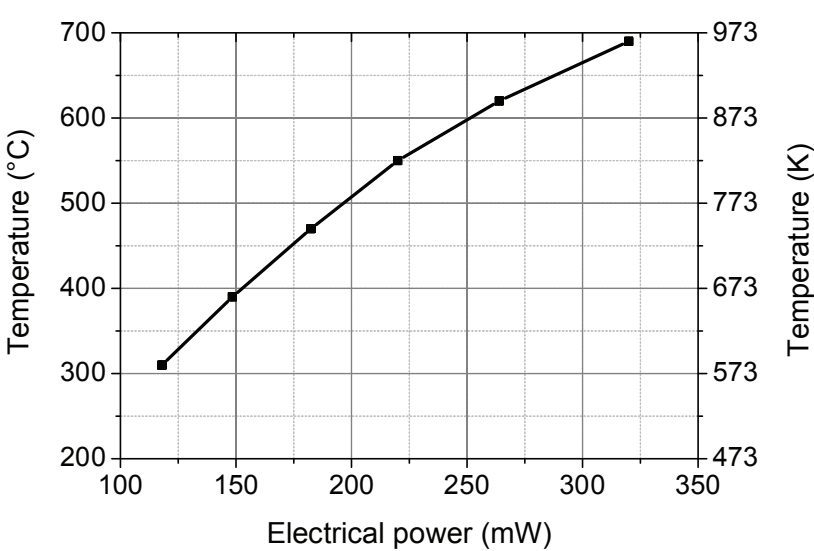

Fig. 7: Measured operating temperature in dependence on the electrical power

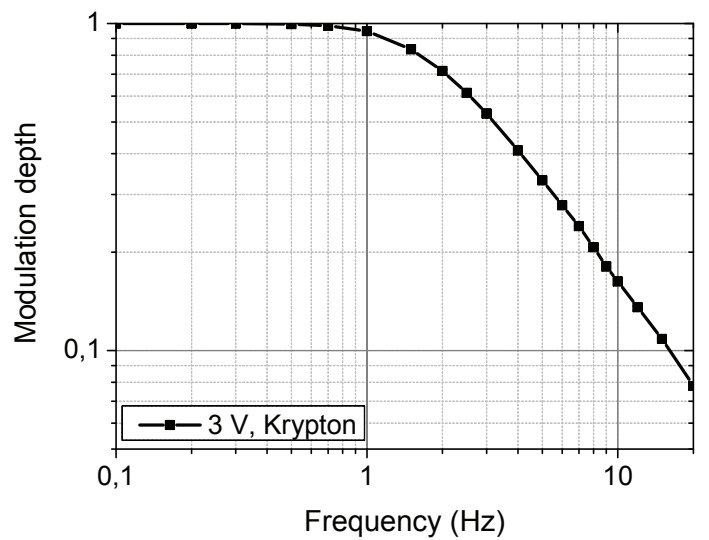

Fig. 8: Measured frequency response of the modulation depth for a constant voltage square-wave drive

The thin and low-mass radiating element allows the IR source to be modulated electrically. This is measured in terms of the modulation depth $m$ which is defined by

$m=\frac{\Delta T}{\Delta T_{\max }} \propto \frac{\Delta \Phi}{\Delta \Phi_{\max }}$. 


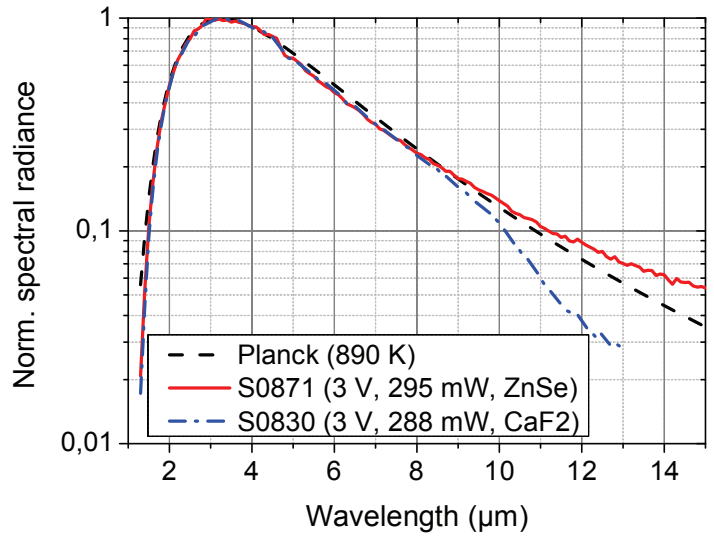

Fig. 9: Comparison of the measured spectral radiance with that of a blackbody radiator of same temperature.

By applying a square-wave voltage the emitter is switched on and off and the temperature amplitude of the radiating element is measured. This is done with a broadband and fast IR detector by measuring the emitted radiant flux $\Phi$ which is proportional to the temperature. With increasing frequency the temperature amplitude decreases due to the thermal mass. Consequently, the frequency response has a low-pass characteristic (Fig. 8). The measurement results show a maximum modulation frequency of about $3 \mathrm{~Hz}$ for $50 \%$ modulation depth. This is sufficient for most applications. But the speed of modulation can be simply increased by a further reduction of the thermal mass.

Finally, the measurement results of the spectral radiance validate the near-blackbody emission characteristic of this thermal infrared source (Fig. 9).

\section{Conclusion and outlook}

In this paper, we presented a novel thermal IR emitter for application in optical gas detection. It features a very high radiant power, nearblackbody emission characteristic and can be modulated electrically. Due to its low thermal mass and the excellent thermal insulation from the heat sink, the electrical power consumption is very low. This enables an application in portable gas sensing devices.

Since the used $\mathrm{NiCr}$ alloy allows a permanent operation at temperatures of up to $1400 \mathrm{~K}$ the fabricated IR source should feature a long-term stable performance and a high life time. Finally, its application in gas analysis will improve the performance and efficiency of gas measurement systems. Further improvements mainly deal with a reduction of the thermal mass of the radiating element in order to increase its modulation depth and modulation frequency.

\section{References}

[1] E. Bakker: Anal. Chem. 76, 3285-3298 (2004); doi: $10.1021 / a c 049580 z$

[2] S. Capone, et al.: J. Optoelec. Adv. Mat. 5, 13351348 (2003); doi: 10.1002/chin.200429283

[3] J. G. Goode, et al.: J. Geophys. Res. 104, 2123721245 (2012); doi:10.1029/1999JD900360

[4] J. R. Howell, R. Siegel, and M. Pinar Menguc: Thermal radiation heat transfer. CRC press (2011); ISBN: 978-1-4398-0533-6

[5] M. Schossig, V. Norkus, G. Gerlach: IEEE Sensors Journal 10, 1564-1565 (2010); doi: 10.1109/JSEN.2010.2046162

[6] M. Schossig: Chapter 18 in: G. Gerlach (Ed.), K.-J. Wolter (Ed.): Bio- and Nanopackaging Techniques for Electron Devices - Advances in Electronic Device Packaging, Heidelberg: Springer, 355-381 (2012); doi: 10.1007/978-3$642-28522-6$ 